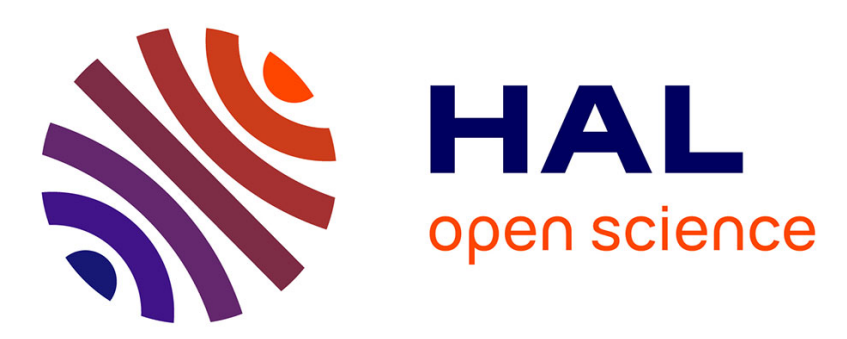

\title{
Dynamic aberration correction for multiharmonic microscopy.
}

Nicolas Olivier, Delphine Débarre, Emmanuel Beaurepaire

\section{To cite this version:}

Nicolas Olivier, Delphine Débarre, Emmanuel Beaurepaire. Dynamic aberration correction for multiharmonic microscopy.. Optics Letters, 2009, 34 (20), pp.3145-7. hal-00681948

\section{HAL Id: hal-00681948 \\ https://hal.science/hal-00681948}

Submitted on 28 Oct 2013

HAL is a multi-disciplinary open access archive for the deposit and dissemination of scientific research documents, whether they are published or not. The documents may come from teaching and research institutions in France or abroad, or from public or private research centers.
L'archive ouverte pluridisciplinaire HAL, est destinée au dépôt et à la diffusion de documents scientifiques de niveau recherche, publiés ou non, émanant des établissements d'enseignement et de recherche français ou étrangers, des laboratoires publics ou privés. 


\title{
Dynamic aberration correction for multiharmonic microscopy
}

\author{
Nicolas Olivier, Delphine Débarre,* and Emmanuel Beaurepaire \\ Laboratory for Optics and Biosciences, Ecole Polytechnique, CNRS, INSERM, F-91128 Palaiseau, France \\ *Corresponding author: delphine.debarre@polytechnique.edu
}

Received July 21, 2009; revised September 8, 2009; accepted September 11, 2009;

posted September 16, 2009 (Doc. ID 114535); published October 8, 2009

\begin{abstract}
We demonstrate image-based aberration correction in a third-harmonic generation (THG) microscope. We describe a robust, mostly sample-independent correction scheme relying on prior measurement of the influence of aberration modes produced by a deformable mirror on the quality of THG images. We find that using image sharpness as an image quality metric, correction of $N$ aberration modes is achieved using $2(2 N+1)$ measurements in a variety of samples. We also report aberration correction in combined multiharmonic and two-photon excited fluorescence experiments. Finally, we demonstrate time-dependent adaptive THG imaging in developing embryonic tissue. (C) 2009 Optical Society of America

OCIS codes: $180.4315,190.4160,110.1080,170.3880,180.6900$.
\end{abstract}

Nonlinear microscopy is commonly used for biological tissue imaging in a variety of contexts. However, aberrations degrade image quality in thick samples, and aberration correction (AC) strategies are being actively investigated [1,2]. Because direct wavefront sensing in situ is nontrivial, image-based modal AC is an attractive possibility [3,4], as shown e.g., for two-photon-excited fluorescence (2PEF) microscopy [5]. The case of image-based AC for coherent nonlinear microscopies such as third-harmonic (TH) generation (THG) [6] or second-harmonic generation (SHG) imaging deserves particular attention, as the harmonic intensity strongly depends on the excitation phase distribution near the focal volume $[7,8]$. Furthermore, the adaptive observation of living biological samples puts constraints on correction strategies, because the acceptable number of measurements used for the correction is limited by sample evolution. In this Letter we develop a robust, efficient AC strategy for THG microscopy, allowing adaptive THG imaging of dynamic tissue.

In modal AC approaches, the aberrated phase is described by a vector $\mathbf{a}=\left\{a_{i}\right\}$ of projections on a basis set of aberration modes $\left\{X_{i}\right\}$. Here we restricted ourselves to the 11 most influential eigenmodes of our deformable mirror (DM) [Fig. 1(b)], excluding tip, tilt, and defocus, and orthogonalized for the Strehl ratio $S$ [9],

$$
\frac{\int \sqrt{I} X_{i} X_{j} \mathrm{~d} \mathbf{r}}{\int \sqrt{I} \mathrm{~d} \mathbf{r}}-\frac{\int \sqrt{I} X_{i} \mathrm{~d} \mathbf{r} \int \sqrt{I} X_{j} \mathrm{~d} \mathbf{r}}{\left(\int \sqrt{I} \mathrm{~d} \mathbf{r}\right)^{2}}=\delta_{i j}
$$

where $I$ is the excitation intensity at the pupil plane of the objective, $\delta_{i j}$ is the Kronecker delta, and the integral is taken over the objective pupil. This choice ensures that low-order aberrations can be properly described, that the correction phase can be accurately produced by the DM, and that for small aberration amplitude, $S \approx 1-|\mathbf{a}|^{2}$. Using this representation, AC requires a limited number of measurements if the influence of each aberration mode on image quality, quantified by a metric $M$, can be described with a single-peaked function that is mostly independent of the sample structure. Such schemes have proven effective for various incoherent microscopy techniques and permit AC in $N$ modes with only $2 N+1$ measurements [3-5].

Owing to the coherent summation of the waves created at different points within the focal volume, the TH signal level results from a complex interplay between sample and field structure [8]; an extended point-spread function can result in a higher signal level [7], and a given amount of aberration may produce sample-dependent intensity variations. Consequently, total image intensity (brightness) is an illdefined quality metric. Instead, it can be expected that a metric reflecting the information content in the image, such as image sharpness (i.e., the sum of the squared pixel values), would be more robust for the design of a sample-independent AC scheme. Indeed, we observed experimentally in all the tested

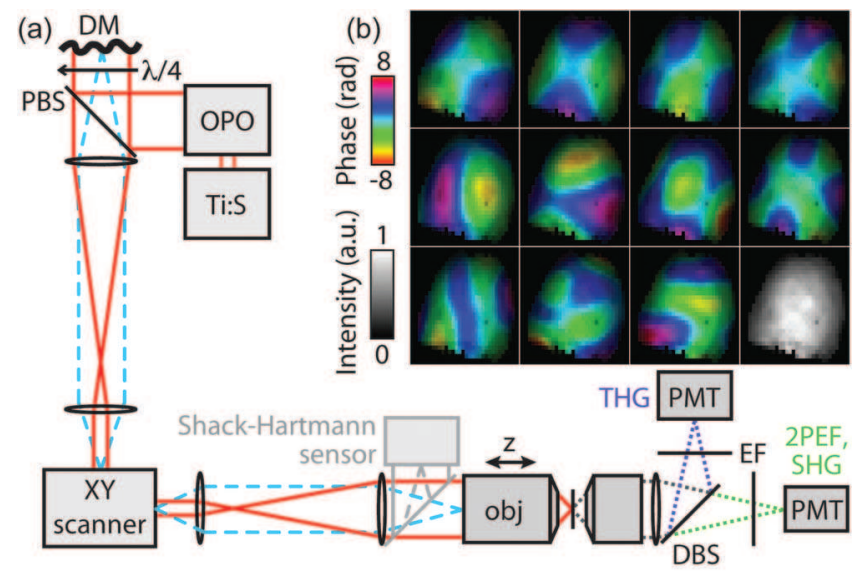

Fig. 1. (a) Experimental setup: $\lambda / 4$, quarter wave plate; PBS, polarizing beamsplitter; DBS, dichroic beamsplitter. Solid lines, excitation path; gray, DM characterization path (not used during AC); dotted lines, emission path; dashed lines, conjugated Fourier planes. (b) Modes $X_{i}$ used for correction, as measured on the $\mathrm{SH}$ sensor. The phase is color coded from purple to red (online) with a brightness proportional to excitation intensity. The last image (lower right) shows the intensity profile alone. 
samples that, unlike total image intensity, image sharpness could be approximated as

$$
M \approx M_{0} \exp \left[-\sum_{i, j} \alpha_{i j} a_{i} a_{j}\right]=M_{0} \exp \left[-\mathbf{a}^{T} \cdot A \cdot \mathbf{a}\right]
$$

where the influence matrix $A=\left\{\alpha_{i j}\right\}$ is symmetric positive-definite (i.e., $M$ reaches its maximum value only in the absence of aberration) and exhibits little sample-dependent variation $(<10 \%)$. In most situations, image sharpness is thus a suitable metric for AC purpose.

The nondiagonal elements of $A, \alpha_{i j}$, describe the variation in the correction in one mode $i$ as a function of the amount of aberration in mode $j$. If the modes have independent influence on $M$ (i.e., $\alpha_{i j}=\delta_{i j}$ ), the optimal correction can be obtained without prior knowledge of $A$ from a set of $2 N+1$ measures of $M$ with different known aberrations introduced with the DM [4]; $M_{0}$ with no introduced aberration, and $\left\{M_{i-}\right\}$ (respectively, $\left\{M_{i+}\right\}$ ) with $-b_{i}$ (respectively, $+b_{i}$ ) aberration added in mode $i$, where $b_{i} \approx 1.5 / \sqrt{\alpha_{i i}}$ is chosen to experimentally optimize correction precision. The correction vector $-\mathbf{a}$ is then calculated as

$$
-\mathbf{a}=\left\{\frac{b_{i}}{2} \frac{\ln M_{i+}-\ln M_{i-}}{2 \ln M_{0}-\ln M_{i-}-\ln M_{i+}}\right\} .
$$

Alternatively, if there is crosstalk between modes, prior knowledge of $A$ is required and $-\mathbf{a}$ is obtained as

$$
-\mathbf{a}=A^{-1}\left\{\alpha_{i i} \frac{b_{i}}{2} \frac{\ln M_{i+}-\ln M_{i-}}{2 \ln M_{0}-\ln M_{i-}-\ln M_{i+}}\right\} .
$$

We implemented this AC scheme on a lab-built microscope $[7,10]$ incorporating a DM and a ShackHartmann (SH) sensor (MIRAO-52 and HASO-3, Imagine Optic), and a $20 \times 0.95 \mathrm{NA}$ water-immersion objective with an underfilled pupil (Olympus) [Fig. 1(a)]. Excitation pulses centered at $1180 \mathrm{~nm}$ were provided by an optical parametric oscillator (APE).

Results on a coverslip-covered fixed elderberry stem slice are shown in Fig. 2. To obtain an AC scheme that is mostly sample independent, we measured $A$ on various samples [Figs. 2, 3(a), and 3(d)] as detailed in [4], and calculated an average [Fig. 2(c)] used for all subsequent experiments. AC with this matrix increases THG brightness but also the lateral and axial resolution, which could not be obtained by increasing the excitation power. As we used an average $A$ not specific to this sample, the AC process converged only after two iterations, thus requiring a total of 46 exposures. Subsequent iterations did not yield further improvement. Albeit, the residual aberration amplitude being reduced for each $\mathrm{AC}$ run, the second run yields significant improvement only for large initial aberration, typically $\mathbf{a}^{T} \cdot A \cdot \mathbf{a} \geqslant 0.3$. Hence for smaller amplitudes, correction does not significantly improve further after more than a single round. In contrast, when assuming a diagonal matrix $A$, iterative corrections yield a vanishing intensity
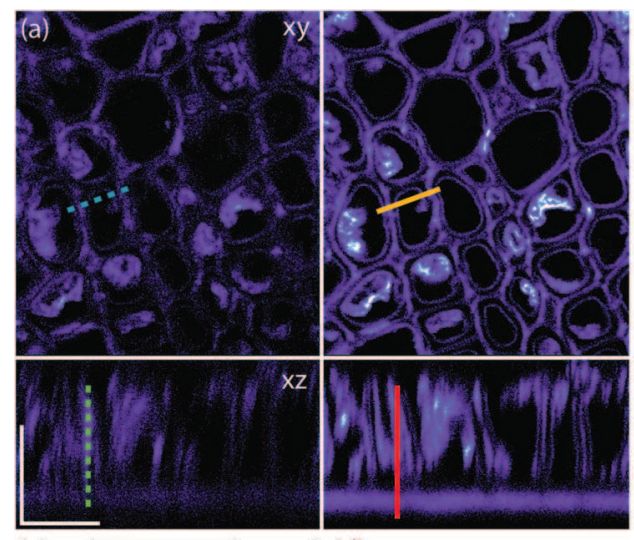

Intensity (a.u.)
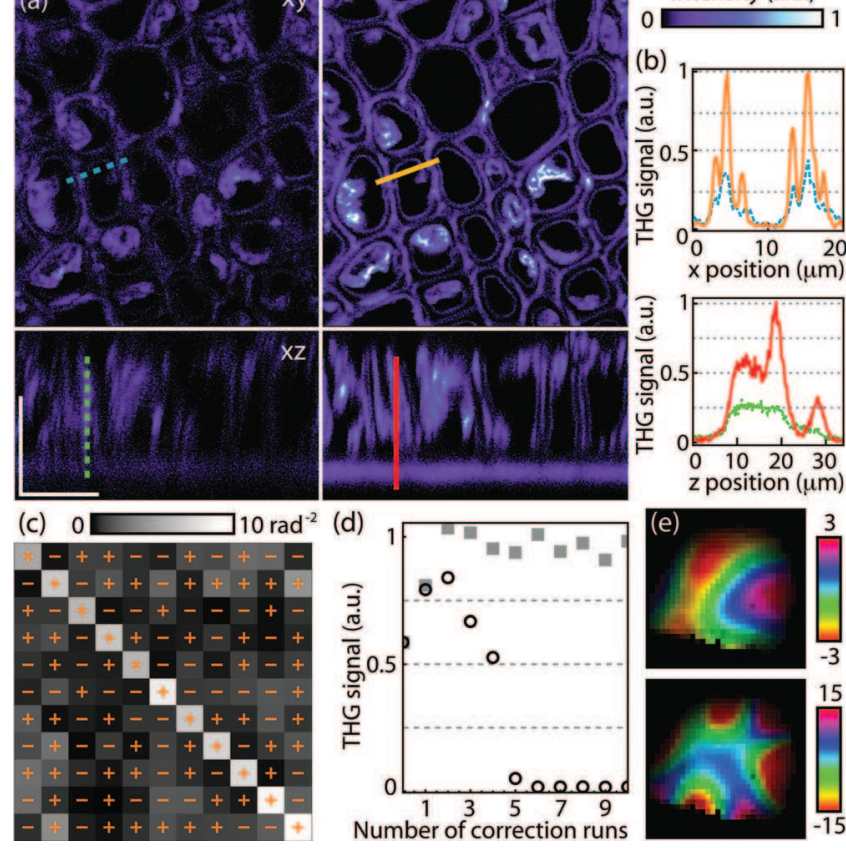

Fig. 2. (a) THG images of an elderberry stem slice (left) before and (right) after $\mathrm{AC}$ on the middle plane of the $z$ stack. Scale bars, $20 \mu \mathrm{m}$. (b) Profiles along the lines in (a). (c) Averaged influence matrix A. (d) TH image brightness as a function of the number of $\mathrm{AC}$ runs, using $A$ (squares) or assuming independent modes (circles). (e) Corresponding final phases (in radians). The top phase was applied for the corrected image in (a).

and an incorrect AC phase [Figs. 2(d) and 2(e)]. This demonstrates that prior knowledge of $A$ is required for accurate AC.

Although the number of images required for complete correction is large, smaller images can be used to perform correction [5]; we found experimentally that for typical signal levels in these images (as low as 10 photons/pixel), the sampling of images could be greatly reduced provided that the sample structure was still visible. Here in all experiments, the scanning speed and the pixel size were multiplied by 4 so that during $\mathrm{AC}$ the imaging time was divided by 16 . The increase in sample exposure due to $\mathrm{AC}$ was thus limited to $\approx 300 \%$ when two rounds of correction were needed and $\approx 150 \%$ for one round.

To test the accuracy of this THG sharpness-based scheme, we performed AC on lily pollen grains producing THG and endogenous $2 \mathrm{PEF}$ signals. Average $2 \mathrm{PEF}$ intensity has been shown theoretically and experimentally to be a suitable metric for sampleindependent $\mathrm{AC}$ [5]. As expected, after two runs of THG-based AC, the correction phase and signal increases were the same as for $2 \mathrm{PEF}$ brightness-based AC [Figs. 3(a) and 3(c)]. Indeed, THG sharpness variations with the amount of aberrations in one mode exhibited a Gaussian shape [see Eq. (2)] with a global maximum at the same position as the $2 \mathrm{PEF}$ brightness curve and without other local maxima [Fig. 3(b) shows the case of mode 1]. THG brightness is also plotted for comparison and would yield incorrect $\mathrm{AC}$ in this case. It should be noted that the $\mathrm{TH}$ signal increase is much greater than that of $2 \mathrm{PEF}$; 

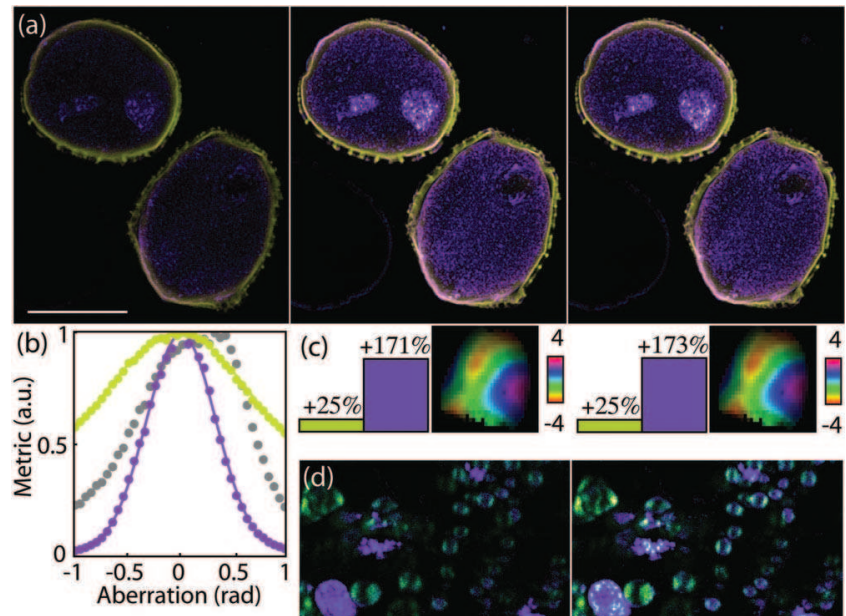

(e)
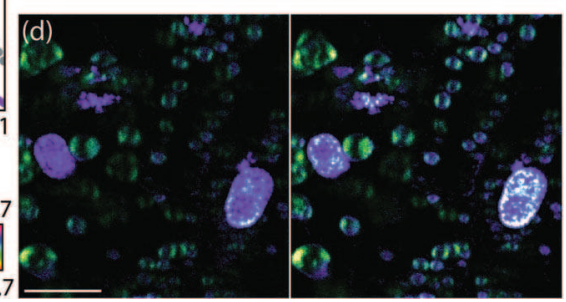

Fig. 3. (a) (Media 1, high resolution; Media 2, low resolution) Combined 2PEF (online)/THG (interior) images of coverslip-covered lily pollen grains, without AC (left), with THG (center), and 2PEF (right) AC. Scale bar, $50 \mu \mathrm{m}$. (b) Metric versus aberration amplitude in mode 1 [see Fig. 1(b)] for THG sharpness (bottom curve), $2 \mathrm{PEF}$ brightness (top curve) and THG brightness (middle curve). Lines, Gaussian fits. (c) Applied phase and 2PEF (left block)/THG (right block) signal increase in (a): left, THG AC; right, 2PEF AC. (d) Combined THG (light)/SHG (dark) images of a coverslip-covered lily anther slice, before (left), and after (right) THG AC. (e) Corresponding AC phase and signal increase. Scale bar, $20 \mu \mathrm{m}$.

this might be due to the coherent nature of THG, for which field summation is affected by the excitation phase distribution. Interestingly, THG-based AC can be used to optimize complex nonlinear signals, such as SHG from starch granules, as shown in Figs. 3(d) and 3(e). The low signal gain compared with Fig. 3(a) stems from the smaller corrected phase amplitude.

Finally we show that, owing to the limited number of needed measurements, our AC scheme can be used on evolving tissue. One application of THG microscopy is the long-term observation of embryo morphogenesis in small organisms [10]. Here we demonstrate time-resolved aberration-corrected imaging of a developing Drosophila embryo at the gastrulation stage. Since correction is updated between each frame, only a small phase needs to be added between successive images so that only one AC run was necessary (except for the first time point, for which two runs were used). Sample illumination was thus multiplied by 2.5 (3.5, including the uncorrected image acquired for comparison) compared to imaging without correction. A development sequence with one corrected frame every minute is shown in Fig. 4 (Media 3 , high resolution; Media 4, low resolution), confirming a significant improvement of both signal and resolution. Note that $\mathrm{AC}$ was achieved here using the matrix $A$ previously measured on other samples, demonstrating that our scheme is mostly sample independant. Interestingly, AC amplitudes in different modes vary during development, indicating that at
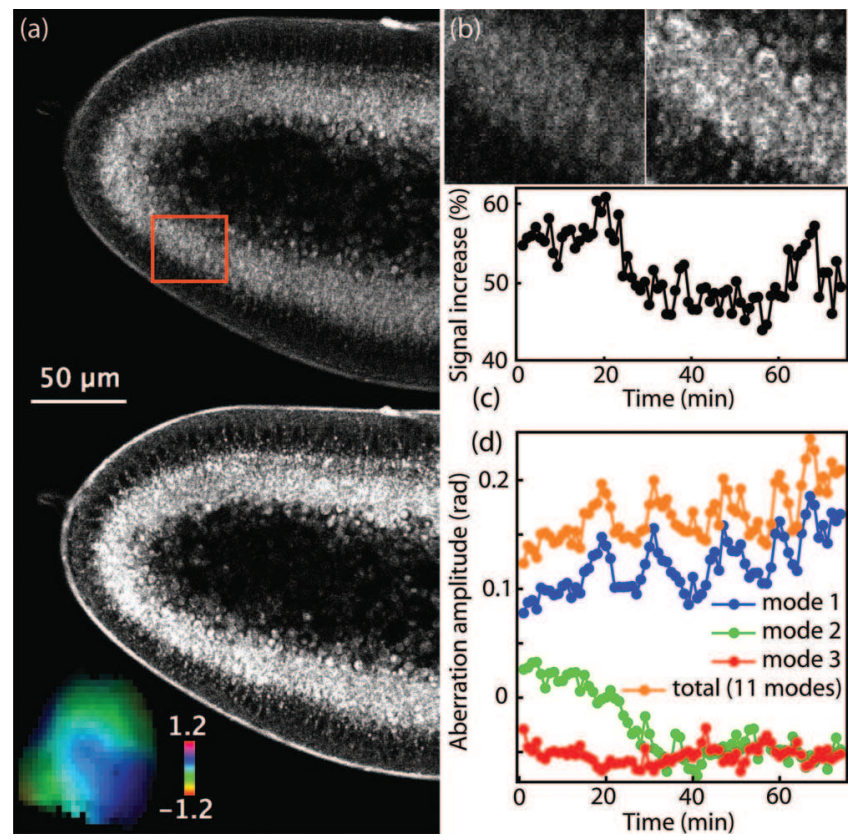

Fig. 4. (a) (Media 3, high resolution; Media 4, low resolutions) Drosophila development imaging without (top) and with (bottom) time-resolved THG-AC. Inset, correction phase (in radians). (b) Zoom on the square in (a). (c) Signal increase after AC. (d) AC amplitude in three modes and total amplitude.

least one part of the aberrations arises from the embryo optical properties. As these can be measured over time, this experiment paves the way for their systematic study in vivo, which would permit optimization of $\mathrm{AC}$ updating frequency.

In conclusion, we proposed an image-based AC scheme suitable for THG-SHG-2PEF microscopy and demonstrated adaptive imaging of a developing embryo. This methodology should generally prove useful in tissue imaging applications.

This work was supported by the Délégation Générale pour l'Armement and by the Agence Nationale de la Recherche (ANR-RIB).

\section{References}

1. P. N. Marsh, D. Burns, and J. M. Girkin, Opt. Express 11, 1123 (2003).

2. M. Rueckel, J. Mack-Bucher, and W. Denk, Proc. Natl. Acad. Sci. USA 103, 17137 (2006).

3. M. J. Booth, M. A. A. Neil, R. Juškaitis, and T. Wilson, Proc. Natl. Acad. Sci. USA 99, 5788 (2002).

4. D. Débarre, E. J. Botcherby, M. J. Booth, and T. Wilson, Opt. Express 16, 9290 (2008).

5. D. Débarre, E. J. Botcherby, T. Watanabe, S. Srinivas, M. J. Booth, and T. Wilson, Opt. Lett. 16, 2495 (2009).

6. Y. Barad, H. Eisenberg, M. Horowitz, and Y. Silberberg, Appl. Phys. Lett. 70, 922 (1997).

7. D. Débarre, W. Supatto, and E. Beaurepaire, Opt. Lett. 30, 2134 (2005).

8. N. Olivier and E. Beaurepaire, Opt. Express 16, 14703 (2008).

9. R. Herloski, J. Opt. Soc. Am. A 2, 1027 (1985).

10. D. Débarre, W. Supatto, E. Farge, B. Moulia, M.-C. Schanne-Klein, and E. Beaurepaire, Opt. Lett. 29, 2881 (2004). 